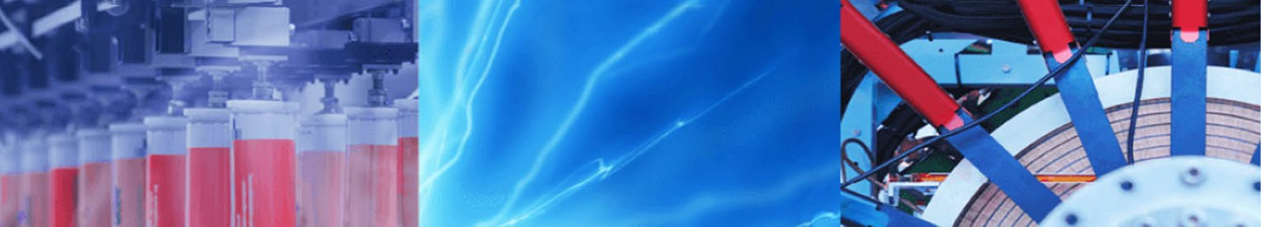

Research Article

\title{
A fast non-searching algorithm for radar maneuvering target detection
}

\author{
Wenchao $\mathrm{Yu}^{1} \cdot$ Weimin $\mathrm{Su}^{2} \cdot$ Hong Gu${ }^{2} \cdot$ Jianchao Yang ${ }^{2} \cdot$ Xingyu $\mathrm{Lu}^{2}$
}

(c) Springer Nature Switzerland AG 2019

\begin{abstract}
The detection of maneuvering targets is a challenging work for radar system. During a long integration time, the target's complex motion will cause severe range migration and Doppler frequency migration, which greatly degrade the energy integration performance. In order to obtain a well-focused result of the maneuvering target, this paper proposes a novel and fast algorithm without any parameter searching process. In our algorithm, a parameter separation (PS) operation is conducted to isolate the target's acceleration from other motion parameters. Then, the second-order keystone transform (SOKT) is utilized to correct range curvature and relocate the target's energy into one range cell. Lastly, the target's energy is integrated along the slow time axis via performing the non-uniform sparse Fourier transform (NUSFT), which is a fast implementation of Fourier transform (FT) for the input signal with unequally sampled points and frequency domain sparse representation. According to the simulation results, the proposed PS-SOKT-NUSFT method can realize multiple targets detection due to its good ability to suppress cross terms. In addition, the runtime of PS-SOKT-NUSFT (106.23 s) is much shorter than the PS-acceleration search (AS) method (2168.67 s).
\end{abstract}

Keywords Radar maneuvering target detection - Parameter separation (PS) - The second-order keystone transform $(\mathrm{SOKT}) \cdot$ Non-uniform sparse Fourier transform (NUSFT)

\section{Introduction}

In the application of radar detection for the moving target, an effective approach for the improvement of the output signal-to-noise ratio (SNR) is prolonging the coherent integration time. However, the complex motion of the target will induce severe range migration and Doppler frequency migration after conducting range compression, which greatly degrade the detection ability of radar [1, 2].

The linear range migration, i.e., range walk, is caused by the uniform motion of the target during a long integration period. In order to remove range walk, some algorithms have been proposed which aim at accumulating the energy along the linear trajectory, such as Radon transform (RT) [3-5], Hough transform (HT) [6-9], Radon Fourier transform (RFT) [10-12] and improved axis rotation MTD
(IAR-MTD) [13]. The above methods need to conduct the parameter searching process which has a high computational burden. In [14], keystone transform (KT) is proposed to rescale the slow time axis and eliminate the coupling between range frequency and slow time. As a linear correction algorithm, KT has a good anti-noise performance and does not require search. In [15], range frequency polynomial-phase transform (RFPPT) is proposed to correct range walk via reducing the order of slow time variable, which has a lower computational complexity than KT.

The target's high-order motions will induce the nonlinear range migration, i.e., range curvature. Meanwhile, Doppler frequency migration will occur and cause the target's energy to spread over multiple Doppler frequency cells. In order to accumulate the target's energy in both range and Doppler dimensions, some brute-force searching algorithms have

$\checkmark$ Weimin Su, wmsu_njust@163.com | ${ }^{1}$ Nanjing Electronic Devices Institute, Nanjing, Jiangsu Province, China. ${ }^{2}$ Nanjing University of Science and Technology, No. 200, Xiaolingwei, Xuanwu District, Nanjing, Jiangsu Province, China.

SN Applied Sciences (2019) 1:1222 | https://doi.org/10.1007/s42452-019-1245-2

Received: 7 February 2019 / Accepted: 10 September 2019 / Published online: 16 September 2019 
been proposed with a heavy computational load, e.g., the generalized Radon Fourier transform (GRFT) [16] and polynomial Radon-polynomial Fourier transform (PRPFT) [17]. In addition, the high-order $\mathrm{KT}$ algorithms without search have been adopted, e.g., the second-order keystone transform (SOKT) [18] and the third-order keystone transform (TOKT) [19]. However, there will be a residual trajectory existing after conducting KT when the maneuvering target has a complex motion, thus some extra operations are needed to compensate the residual range migration. In [20], the parameter separation (PS)-acceleration search (AS) approach is proposed for target focusing, it only needs to search for the target's acceleration after isolating it from other motion parameters.

Improved from PS-AS, a novel maneuvering target detection algorithm is proposed in this paper. The main contributions of this work are presented as follows:

1. Through theoretical analysis, it is demonstrated that the proposed method has no need to search for the target's motion parameters, thus a lower computational cost can be obtained compared with the traditional methods based on brute-force search.

2. The non-uniform sparse Fourier transform (NUSFT) has been proposed, which is the combination of nonuniform fast Fourier transform (NUFFT) [21-24] and sparse Fourier transform (SFT) [25].

3. The multiple targets detection ability of the proposed method has been analyzed.

4. The good detection performance of the proposed method has been validated via the results of some simulation experiments.

The outline of this paper is organized as follows. Section 2 gives the signal model of radar maneuvering target detection. Section 3 introduces the proposed method for mono- and multi- targets detection. Section 4 gives the computational complexity analysis. Section 5 shows the results of some simulation experiments. Finally, the conclusion is drawn in Sect. 6.

\section{Signal model}

Suppose that a radar transmits LFM pulses, where the number of coherent integrated pulses is $M$, the pulse repetition interval is $T_{r}$. The transmitted signal has the following form:

$s_{t}\left(t_{m}, t\right)=\operatorname{rect}\left(\frac{t}{T_{p}}\right) \exp \left(j \pi \mu t^{2}\right) \exp \left(j 2 \pi f_{c} t_{m}\right)$ $\ln (1), t$ is the fast time, $t_{m}=m T_{r}$ is the slow time, where $m=-\frac{M-1}{2}, \ldots,-1,0,1, \ldots, \frac{M-1}{2}, M$ is assumed to be an odd integer. $T_{p}, f_{c}, \mu$ and $\operatorname{rect}(\cdot)$ represent the pulse duration, carrier frequency, chirp rate and rectangle window, respectively.

The instantaneous slant range between radar and the maneuvering target can be represented as

$R\left(t_{m}\right)=R_{0}+c_{1} t_{m}+c_{2} t_{m}^{2}+c_{3} t_{m}^{3}$

where $R_{0}, c_{1}, c_{2}$ and $c_{3}$ denote the initial slant range, radial velocity, acceleration and jerk of the target, respectively.

The received baseband signal is expressed as

$$
\begin{aligned}
& s_{r}\left(t_{m}, t\right)=A_{1} \text { rect }\left[\frac{t-2 R\left(t_{m}\right) / c}{T_{p}}\right] \\
& \exp \left[j \pi \mu\left(t-\frac{2 R\left(t_{m}\right)}{c}\right)^{2}\right] \exp \left[-j \frac{4 \pi}{\lambda} R\left(t_{m}\right)\right]
\end{aligned}
$$

where $A_{1}$ denotes the signal amplitude, $\lambda=\frac{c}{f_{c}}$ is the wavelength of the transmitted signal, $c$ denotes the light speed.

After performing pulse compression, the signal in the range frequency domain can be expressed as

$S_{c}\left(t_{m}, f\right)=A_{2} \operatorname{rect}\left(\frac{f}{B}\right) \exp \left[-j \frac{4 \pi}{c}\left(f+f_{c}\right)\left(R_{0}+c_{1} t_{m}+c_{2} t_{m}^{2}+c_{3} t_{m}^{3}\right)\right]$

where $A_{2}$ denotes the signal amplitude, $f$ is the range frequency variable, $B=\mu T_{p}$ is the bandwidth of the transmitted signal.

After performing the range inverse fast Fourier transform (IFFT), the compressed signal in the range time domain is obtained as

$$
\begin{aligned}
s_{c}\left(t_{m}, t\right)= & A_{3} \operatorname{sinc}\left\{B\left[t-\frac{2}{c}\left(R_{0}+c_{1} t_{m}+c_{2} t_{m}^{2}+c_{3} t_{m}^{3}\right)\right]\right\} \\
& \times \exp \left[-j \frac{4 \pi}{\lambda}\left(R_{0}+c_{1} t_{m}+c_{2} t_{m}^{2}+c_{3} t_{m}^{3}\right)\right]
\end{aligned}
$$

where $A_{3}$ denotes the signal amplitude after performing range IFFT. From (5), one can see that $c_{1}$ will cause range walk and Doppler center shift, while $c_{2}$ and $c_{3}$ will cause range curvature and Doppler frequency migration. During a long integration time, both range migration and Doppler frequency migration will deteriorate the target detection performance. In the next section, we will propose an efficient algorithm to overcome the above problems and accumulate the target's energy coherently. 


\section{Coherent integration via PS-SOKT-NUSFT}

\subsection{Mono-target detection}

\subsubsection{Range migration correction}

According to the aforementioned signal model, it can be seen that the slow time axis is symmetric around $t_{m}=0$, then the reversed slow time variable $\overleftarrow{t_{m}}$ can be expressed as below

$\overleftarrow{t_{m}}=-m T_{r}\left(m=-\frac{M-1}{2}, \ldots,-1,0,1, \ldots, \frac{M-1}{2}\right)$

Inserting (6) into (4), we obtain the slow time reversed form of the range compressed signal, i.e., where $t_{n}$ represents the scaled slow time after performing SOKT.

Inserting (11) into (8) and performing range IFFT, we obtain

$s_{2}\left(t_{n}, t\right)=A_{5} \operatorname{sinc}\left[B\left(t-\frac{4 R_{0}}{c}\right)\right] \exp \left(-j \frac{8 \pi}{\lambda} c_{2} t_{n}^{2}\right)$

where $A_{5}$ denotes the signal amplitude after performing range IFFT.

From (12), one can see that no range migration remains after conducting SOKT, thus the target's energy is located in one fixed range cell. However, there is still Doppler frequency migration to be corrected in the slow time dimension.

$S_{c}\left(\overleftarrow{t_{m}}, f\right)=A_{2} \operatorname{rect}\left(\frac{f}{B}\right) \exp \left[-j \frac{4 \pi}{c}\left(f+f_{c}\right)\left(R_{0}+c_{1}{\overleftarrow{t_{m}}}+c_{2}{\overleftarrow{t_{m}}}^{2}+c_{3}{\overleftarrow{t_{m}}}^{3}\right)\right]$

Then, the PS operation is defined as [20]

$$
\begin{aligned}
S_{1}\left(t_{m^{\prime}} f\right)= & S_{c}\left(t_{m^{\prime}}, f\right) S_{c}\left(\overleftarrow{t_{m}} f\right) \\
= & A_{2}^{2} \operatorname{rect}\left(\frac{f}{B}\right) \exp \left[-j \frac{8 \pi}{c}\left(f+f_{c}\right) R_{0}\right] \\
& \times \exp \left\{-j \frac{4 \pi}{c}\left(f+f_{c}\right)\left[c_{1}(m-m) T_{r}\right]\right\} \\
& \times \exp \left\{-j \frac{4 \pi}{c}\left(f+f_{c}\right)\left[c_{2}\left(m^{2}+m^{2}\right) T_{r}^{2}\right]\right\} \\
& \times \exp \left\{-j \frac{4 \pi}{c}\left(f+f_{c}\right)\left[c_{3}\left(m^{3}-m^{3}\right) T_{r}^{3}\right]\right\} \\
= & A_{2}^{2} \operatorname{rect}\left(\frac{f}{B}\right) \exp \left[-j \frac{8 \pi}{c}\left(f+f_{c}\right)\left(R_{0}+c_{2} t_{m}^{2}\right)\right]
\end{aligned}
$$

The expression of (8) in the range time domain is written as below

$s_{1}\left(t_{m}, t\right)=A_{4} \operatorname{sinc}\left\{B\left[t-\frac{4}{c}\left(R_{0}+c_{2} t_{m}^{2}\right)\right]\right\} \exp \left[-j \frac{8 \pi}{\lambda}\left(R_{0}+c_{2} t_{m}^{2}\right)\right]$

where $A_{4}$ denotes the signal amplitude.

In (9), it can be seen that the effect of target's velocity and jerk has been removed, whereas there is still a trajectory existing, i.e.,

$R^{\prime}\left(t_{m}\right)=2\left(R_{0}+c_{2} t_{m}^{2}\right)$

In order to correct the residual range curvature, SOKT is conducted to remove the coupling between $f$ and $t_{m^{\prime}}^{2}$ i.e.,

$t_{m}=\left(\frac{f_{c}}{f+f_{c}}\right)^{1 / 2} t_{n}$

\subsubsection{Coherent integration via NUSFT}

In (12), the extracted signal at $t=\frac{4 R_{0}}{c}$ is modeled as a LFM signal with zero centroid frequency, thus a direct fast Fourier transform (FFT) performed along the slow time axis is invalid because the sampling points are non-uniform. In order to realize coherent integration, we perform FT with respect to $t_{n}^{2}$ as below

$$
\begin{aligned}
S_{3}\left(f_{n}, t\right) & =\int s_{2}\left(t_{n}, t\right) \exp \left(-j 2 \pi f_{n} t_{n}^{2}\right) d t_{n}^{2} \\
& =A_{6} \operatorname{sinc}\left[B\left(t-\frac{4 R_{0}}{c}\right)\right] \operatorname{sinc}\left(f_{n}+\frac{4 c_{2}}{\lambda}\right)
\end{aligned}
$$

where $f_{n}$ represents the frequency variable with respect to $t_{n}, A_{6}$ denotes the signal amplitude after conducting $\mathrm{FT}$.

It can be seen that in (13) that the signal's energy is accumulated as a solo peak and the target can be detected. In realistic applications, performing non-uniform discrete Fourier transform (NUDFT) to realize FT needs a large number of complex multiplications which are computationally expensive. For the sake of improving efficiency, the existing NUFFT algorithm can be adopted to fulfill FT of the non-uniformly sampled signal, which has the same computational complexity as FFT [21-24]. Thus, the slow time FT conducted in (13) can be implemented via NUFFT which is faster than DFT. Here, noticing that the frequency domain output is sparse since $M \gg 1$, thus the integration process can be further accelerated via replacing the FFT in NUFFT by a faster spectrum analysis tool, i.e., SFT [25]. Below, the procedures of the the proposed NUSFT algorithm are given. 
Suppose that the input data $s_{n}$ is sampled at non-uniform points $x_{n}$ with the length of $M$ which is equal to the length of output signal. Set the oversampling factor and interpolation length as $p$ and $L$, respectively. Select Kaiser-Bessel window as below [24]

$\phi(x)=\sqrt{\frac{2}{\pi}} \frac{\sinh \left(\alpha \sqrt{L^{2}-x^{2}}\right)}{\sqrt{L^{2}-x^{2}}}$
$\phi(\xi)= \begin{cases}I_{0}\left(L \sqrt{\alpha^{2}-\xi^{2}}\right), & |\xi| \leq \alpha \\ 0, & |\xi|>\alpha\end{cases}$

where $\alpha=\pi\left(2-\frac{1}{p}\right)-0.01$ is the width of the window function.

Conduct the following steps:

1. Calculate the Fourier coefficients:

$u_{\mu_{n}+l}=\sum_{n=1}^{M} \sum_{l=-L}^{L} \frac{1}{\sqrt{2 \pi}} \phi\left(p x_{n}-\mu_{n}-I\right) s_{n}$

where $\mu_{n}$ is a vector which is the nearest integer to $p x_{n}$.

2. Compute the SFT of $u_{j}$ :

$U_{j}=\operatorname{SFT}\left(u_{j}\right)$

where $j=-\frac{p M}{2},-\frac{p M}{2}+1, \ldots, \frac{p M}{2}-1$.

3. Scale the values to obtain the NUSFT output:

$S_{n}=\frac{U_{n}}{\phi_{n}}$

where $n=-\frac{M}{2},-\frac{M}{2}+1, \ldots, \frac{M}{2}-1$.

It should be noted that in (17), SFT is conducted to replace the $p M$-points FFT which is performed in NUFFT. Set the sparsity parameter in NUSFT as 1 and under the condition that $p \ll M$, the computational complexities of NUSFT, NUFFT and NUDFT are, respectively, $O\left(\sqrt{N \log _{2} N} \log _{2} N\right), O\left(N \log _{2} N\right)$, and $O\left(N^{2}\right)$, thus it is evident that NUSFT can realize energy integration with the lowest computational cost.

\subsection{Multi-targets detection}

In the aforementioned analysis, the effectiveness of the proposed method for mono-target detection has been demonstrated. Nevertheless, there might be more than one target for radar to detect in practical situations, thus it is necessary to discuss the ability of PS-SOKT-NUSFT to suppress cross terms. Note that the number of targets is $K$, then the compressed signal in the range frequency domain can be expressed as

$$
\begin{aligned}
S_{c}\left(t_{m}, f\right)= & \sum_{i=1}^{K} A_{2, i} \operatorname{rect}\left(\frac{f}{B}\right) \\
& \times \exp \left[-j \frac{4 \pi}{c}\left(f+f_{c}\right)\left(R_{0, i}+c_{1, i} t_{m}+c_{2, i} t_{m}^{2}+c_{3, i} t_{m}^{3}\right)\right]
\end{aligned}
$$

where $R_{0, i}, c_{1, i}, c_{2, i}$ and $c_{3, i}$ represent, respectively, the initial slant range, radial velocity, acceleration and jerk of the $i$ th target. After performing PS and SOKT on $S_{c}\left(t_{m}, f\right)$, we obtain the compressed signal in the range time domain, i.e.,

$$
\begin{aligned}
s_{2}\left(t_{n}, t\right)= & s_{2, \text { self }}\left(t_{n}, t\right)+s_{2, \text { cross }}\left(t_{n,} t\right) \\
= & \sum_{i=1}^{K} A_{5, i} \operatorname{sinc}\left[B\left(t-\frac{4 R_{0, i}}{c}\right)\right] \exp \left(-j \frac{8 \pi}{\lambda} c_{2, i} t_{n}^{2}\right) \\
& +\sum_{i=1}^{K} \sum_{j=1, j \neq i}^{K} A_{5, i j} \operatorname{sinc}\left[B\left(t-\tau_{i j}\right)\right] \\
& \times \exp \left[-j \frac{4 \pi}{\lambda}\left(\nabla R_{0, i j}+\Delta c_{1, i j} t_{n}+\nabla c_{2, i j} t_{n}^{2}+\Delta c_{3, i j} t_{n}^{3}\right)\right]
\end{aligned}
$$

where

$\tau_{i j}=\frac{2 \nabla R_{0, i j}+\Delta c_{1, i j} t_{n}-\Delta c_{3, i j} t_{n}^{3}}{c}$

$\nabla R_{0, i j}=R_{0, i}+R_{0, j}$

$\Delta c_{1, i j}=c_{1, i}-c_{1, j}$

$\nabla c_{2, i j}=c_{2, i}+c_{2, j}$

$\Delta c_{3, i j}=c_{3, i}-c_{3, j}$

From (20) to (25), it is obvious that after conducting PS and SOKT, the energy of each self term has been relocated in one range cell, whereas range migration still exists for cross terms. What's more, the first- and thirdorder phase terms will affect the focusing performance of cross terms in the slow time domain. Therefore, the energy of cross terms will be suppressed while each self term will generate as a solo peak after conducting slow time NUSFT.

The detailed procedures of the proposed method are shown as below. 


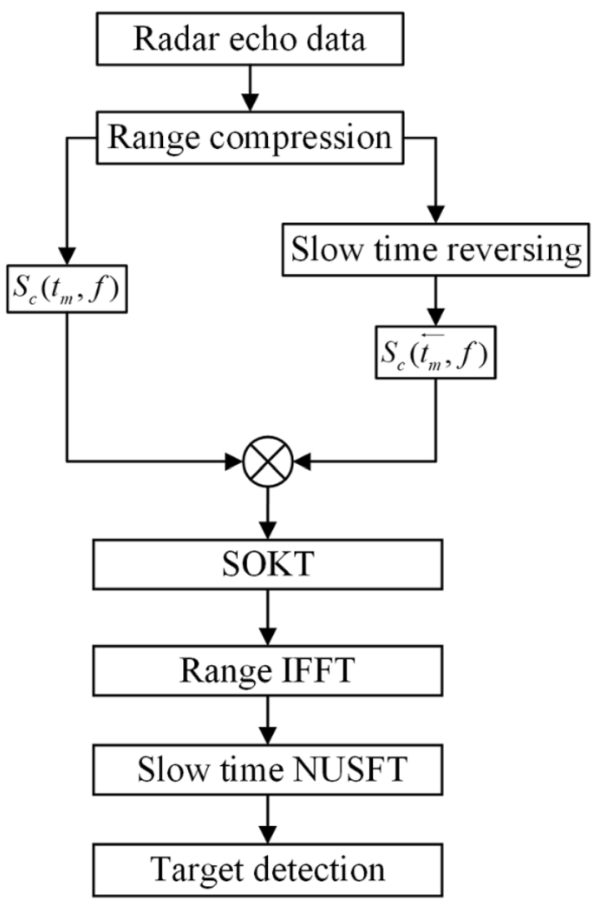

Fig. 1 Flowchart of the proposed method

1. Perform pulse compression of the radar echo data and obtain the signal $S_{c}\left(t_{m}, f\right)$ in the range frequency domain.

2. Multiply $S_{c}\left(t_{m}, f\right)$ with its slow time reversed result $S_{c}\left(\overleftarrow{t_{m}}, f\right)$ to remove the effect of velocity and jerk.

3. Conduct SOKT and range IFFT to correct residual range curvature.

4. Carry out NUSFT along the slow time axis to fulfill coherent integration and detect the target.

The flowchart of the proposed algorithm is shown in Fig. 1.

\section{Computational complexity analysis}

In this section, the computational complexity of the proposed method is analyzed. Denote that the number of range cells and integrated pulses are $N$ and $M$, respectively. The number of large frequency coefficients in

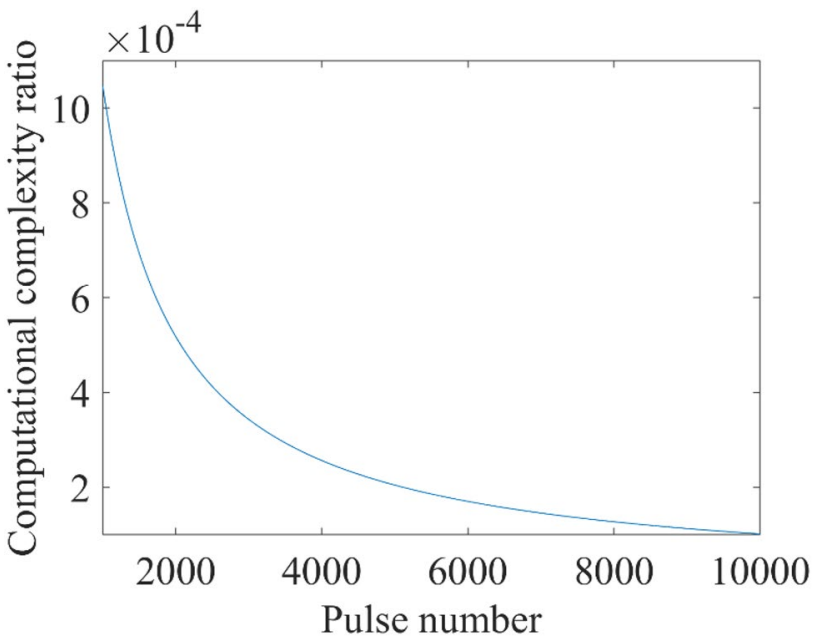

Fig. 2 Computational complexity ratio

NUSFT is set as 1 . For the PS operation which needs a 2-D complex multiplication, the computational complexity is $O(N M)$. Subsequently, the chirp-z transform (CZT) based SOKT and range IFFT are performed with the computational complexity of $O\left[N M\left(\log _{2} N+\log _{2} M\right)\right]$. Lastly, the slow time NUSFT is conducted with the computational complexity of $O\left(N \sqrt{M \log _{2} M} \log _{2} M\right)$. As for the the energy accumulation process in the PS-AS algorithm [20], a 2-D matched filtering process is conducted in each search for acceleration, which has the computational complexity of $O\left[N_{s 2} N M\left(1+\log _{2} N+\log _{2} M\right)\right]$, where $N_{s 2}$ represents the searching times of acceleration. For comparison, Table 1 shows the computational complexities of PS-SOKT-NUSFT and PS-AS. Define the the computational complexity ratio as the complexity metric of PS-SOKT-NUSFT divided by that of PS-AS, Fig. 2 depicts the curve of computational complexity ratio against pulse number under the assumption that $N=M=N_{s 2}$. It is evident that with the increase of pulse number, PS-SOKT-NUSFT has a much lower computational complexity than PS-AS, due to its two advantages: (1) It has no need to search for the target's motion parameters; (2) It applies NUSFT instead of NUDFT to speed up the coherent integration process.

Table 1 Computational complexities

\begin{tabular}{ll}
\hline Method & Computational complexity \\
\hline PS-SOKT-NUSFT & $O(N M)+O\left[N M\left(\log _{2} N+\log _{2} M\right)\right]+O\left(N \sqrt{M \log _{2} M} \log _{2} M\right)$ \\
PS-AS & $O(N M)+O\left[N_{s 2} N M\left(1+\log _{2} N+\log _{2} M\right)\right]$ \\
\hline
\end{tabular}


Table 2 Radar system parameters

\begin{tabular}{ll}
\hline Carrier frequency & $0.5 \mathrm{GHz}$ \\
Bandwidth & $15 \mathrm{MHz}$ \\
Sample frequency & $30 \mathrm{MHz}$ \\
Pulse repetition frequency & $1000 \mathrm{~Hz}$ \\
Pulse duration & $10 \mu \mathrm{s}$ \\
Pulse Number & 2049 \\
\hline
\end{tabular}

\section{Numerical experiments and performance analysis}

In this section, several simulation experiments are conducted to validate effectiveness of the proposed method. Radar system parameters are listed in Table 2, complex white Gaussian noise is added to the radar echo signal with the input SNR set as $-3 \mathrm{~dB}$.

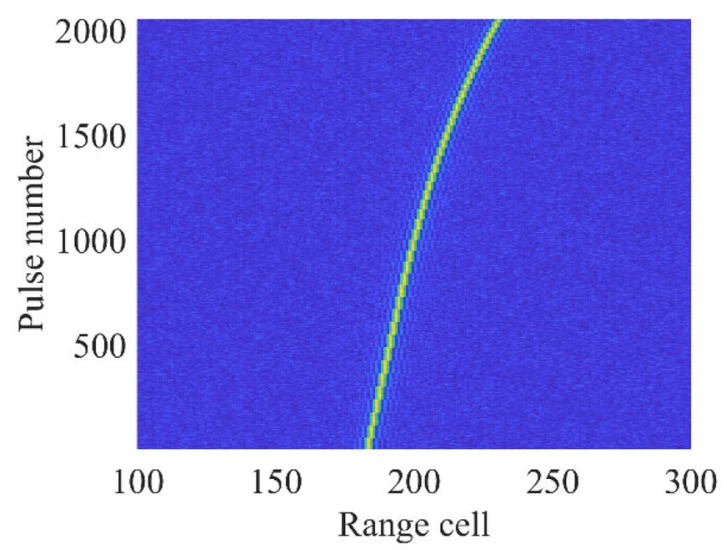

a

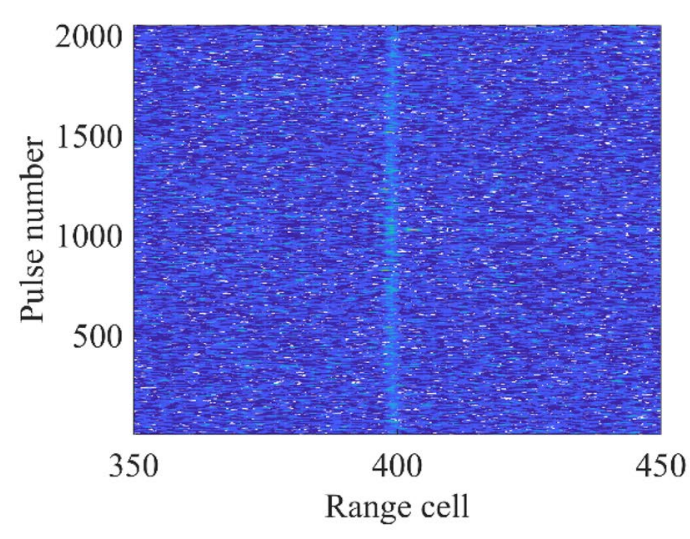

C

\subsection{Proposed method with mono-target detection}

Consider a maneuvering target which has the motion parameters of $c_{1}=100 \mathrm{~m} / \mathrm{s}, c_{2}=30 \mathrm{~m} / \mathrm{s}^{2}$ and $c_{3}=15 \mathrm{~m} / \mathrm{s}^{3}$, respectively. At $t_{m}=0$, the target is located at the 200th range cell. Figure 3a shows the target's trajectory after performing range compression, it can be seen that severe range migration occurs due to the long time complex motion of the target. Figure 3b gives the target's trajectory after conducting the PS operation, it is observed that only the symmetric range curvature caused by acceleration exists. According to (2) and (10), it can be calculated that the migrated range cells before and after conducting PS operation are, respectively, 183th $\rightarrow$ 200th $\rightarrow$ 230th and 413th $\rightarrow$ 400th $\rightarrow$ 413th.

Figure $3 c$ shows the result after performing SOKT, it is evident that the range curvature has been corrected effectively. Finally, the target is well focused via carrying out the slow time NUSFT, as shown in Fig. $3 d$.

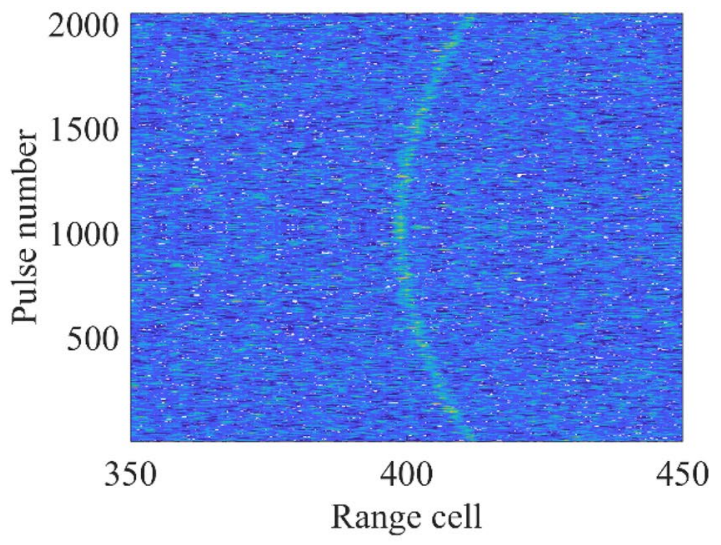

b

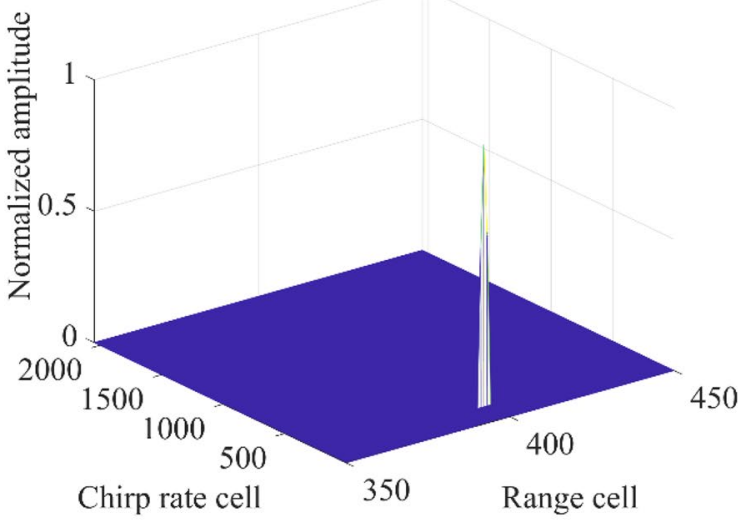

d

Fig. 3 Simulation results of a single maneuvering target. a Target's trajectory after performing range compression. b Target's trajectory after performing the PS operation. c Target's trajectory after performing SOKT. d Focused result of slow time NUSFT 
Fig. 4 Simulation results of two maneuvering targets. a Target's trajectory after performing range compression. b Target's trajectory after performing the PS operation. c Target's trajectory after performing SOKT. d Focused result of slow time NUSFT. e Vertical view of slow time NUSFT. $\mathbf{f}$ Vertical view of slow time NUFFT
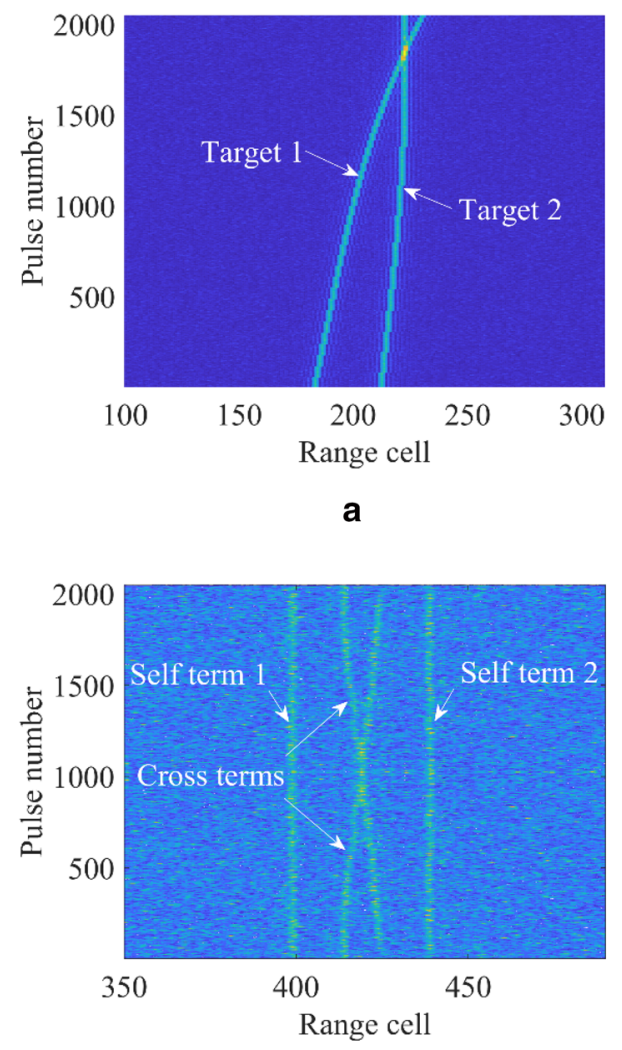

C

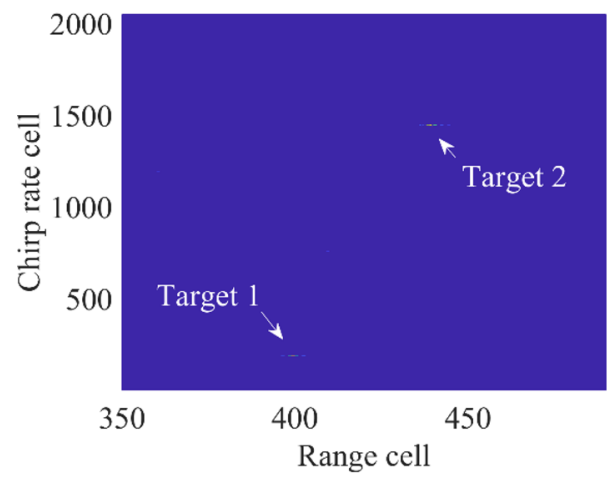

e

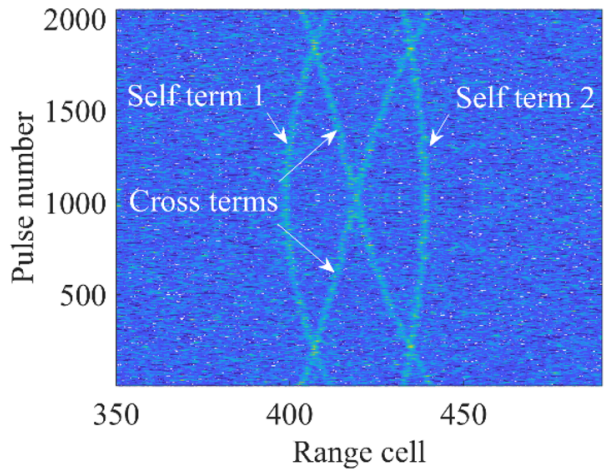

b

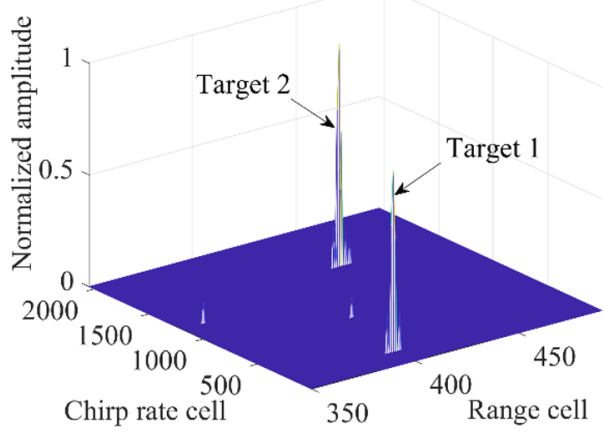

d

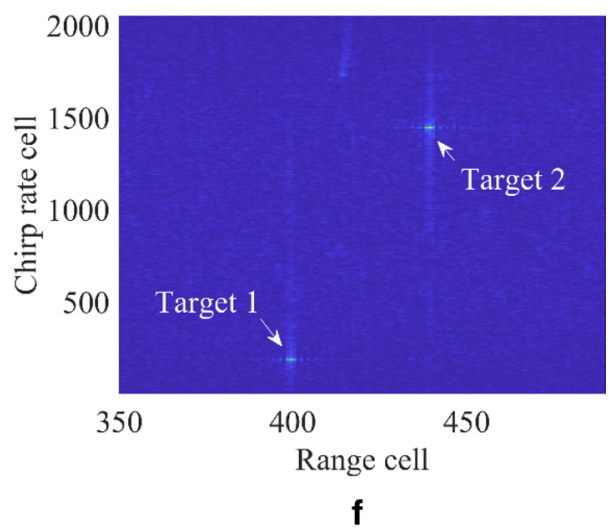

\subsection{Proposed method with multi-targets detection}

In this section, the radar is used to detect two maneuvering targets. The motion parameters of target 1 are the same as that in Sect. 5.1, the motion parameters of target 2 are $c_{1}=30 \mathrm{~m} / \mathrm{s}, c_{2}=-15 \mathrm{~m} / \mathrm{s}^{2}$ and $c_{3}=-5 \mathrm{~m} / \mathrm{s}^{3}$, respectively. At $t_{m}=0$, target 2 is located at the 220th range cell. The result of range compression is shown in Fig. $4 \mathrm{a}$, where severe range migration occurs for both targets. Figure $4 \mathrm{~b}$ gives the result after performing PS, it can be seen that two trajectories of cross terms exist in addition to that of self terms. Subsequently, SOKT is conducted and the result is shown in Fig. $4 c$, it is evident that the range curvature of self terms has been removed, while range migration still remains for cross terms. Lastly, slow time NUSFT is conducted to accumulate the energy of self terms and detect the targets, as shown in Fig. $4 \mathrm{~d}$. For comparison, Fig. $4 \mathrm{e}$ and $\mathrm{f}$ give the vertical views of the proposed method which is implemented by NUSFT and NUFFT, respectively. It can be seen that NUFFT outputs all the accumulated coefficients, whereas NUSFT only outputs the large frequency coefficients while setting the small coefficients (including the outputs of noise and cross terms) as 0. 


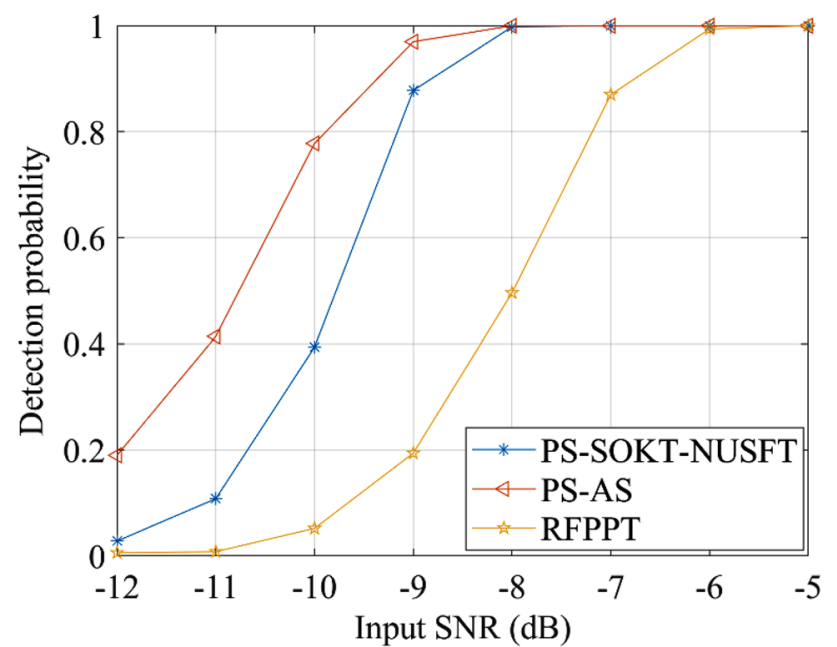

Fig. 5 Detection probability comparison

Table 3 Runtime comparison

\begin{tabular}{lc}
\hline Method & Runtime (s) \\
\hline PS-SOKT-NUSFT & 106.23 \\
PS-AS & 2168.67 \\
\hline
\end{tabular}

\subsection{Detection performance and runtime}

In this section, the detection performance of PS-SOKT-NUSFT is compared with PS-AS and RFPPT. The number of searches in PS-AS is set as 1000 . For each input SNR, 500 Monte Carlo experiments are conducted. Figure 5 shows the curves of detection probability against input SNR for the three methods. It can be seen that as a coherent integration method, PS-SOKT-NUSFT outperforms RFPPT which neglects the target's high-order motion. However, PS-SOKT-NUSFT suffers a slight performance loss compared with PS-AS due to the effect of interpolation and subsampled FFT in NUSFT. Each coin has its two sides, according to the runtime comparison in Table 3, it is evident that PS-SOKT-NUSFT has a much higher efficiency than PS-AS.

\section{Conclusion}

This paper focuses on the topic of radar maneuvering target detection and proposes a fast algorithm to correct range migration and Doppler frequency migration. The proposed method includes three steps: PS, SOKT and slow time NUSFT, which can realize acceleration isolation, range curvature correction and coherent integration, respectively. As a non-searching method, PS-SOKT-NUSFT can avoid the 4-D brute-force search which is widely adopted in the traditional methods such as GRFT and PRPFT. The simulation experiments have validated the effectiveness of the proposed method in multiple targets detection. In addition, the runtime of PS-SOKT-NUSFT and PS-AS in the numerical experiment are, respectively, $106.23 \mathrm{~s}$ and $2168.67 \mathrm{~s}$, which indicates that PS-SOKT-NUSFT has a higher efficiency due to its non-searching process and NUSFT operation. Since the target's highest motion order considered in PS-SOKT-NUSFT is 3, the future work might concern the fast detection of the target with a higher order motion model. Besides, it might be of interest to research the algorithm under the clutter or interference background.

Funding This study was funded by National Natural Science Foundation of China (Grant Numbers: 61471198 and 61671246). This study was also funded by Natural Science Foundation of Jiangsu Province (Grant Numbers: BK20160847 and BK20170855).

\section{Compliance with ethical standards}

Conflict of interest The authors declare that they have no conflict of interest.

\section{References}

1. Li X, Cui G, Yi W, Kong L (2015) An efficient coherent integration method for maneuvering target detection. In: IET international radar conference 2015, 14-16 October 2015 , pp 1-6 https://doi. org/10.1049/cp.2015.1214

2. Yu W, Su W, Gu H (2017) Ground moving target motion parameter estimation using Radon modified Lv's distribution. Digit Signal Process 69:212-223. https://doi.org/10.1016/j. dsp.2017.07.005

3. Fiddy MA (1985) The Radon transform and some of its applications. Opt Acta Int J Opt 32(1):3-4

4. Beylkin G (1987) Discrete Radon transform. IEEE Trans Acoust Speech Signal Process 35(2):162-172. https://doi.org/10.1109/ TASSP.1987.1165108

5. Zhang X, Liao G, Zhu S, Zeng C, Shu Y (2015) Geometry-information-aided efficient radial velocity estimation for moving target imaging and location based on Radon transform. IEEE Trans Geosci Remote Sens 53(2):1105-1117. https://doi.org/10.1109/ TGRS.2014.2334322

6. Carlson BD, Evans ED, Wilson SL (1994) Search radar detection and track with the Hough transform. I. system concept. IEEE Trans Aerosp Electron Syst 30(1):102-108. https://doi. org/10.1109/7.250410

7. Carlson BD, Evans ED, Wilson SL (1994) Search radar detection and track with the Hough transform. II. detection statistics. IEEE Trans Aerosp Electron Syst 30(1):109-115. https://doi. org/10.1109/7.250411

8. Carlson BD, Evans ED, Wilson SL (1994) Search radar detection and track with the Hough transform. III. detection performance 
with binary integration. IEEE Trans Aerosp Electron Syst 30(1):116-125. https://doi.org/10.1109/7.250412

9. Oveis AH, Sebt MA (2017) Coherent method for ground-moving target indication and velocity estimation using Hough transform. IET Radar Sonar Navig 11(4):646-655. https://doi. org/10.1049/iet-rsn.2016.0262

10. Xu J, Yu J, Peng YN, Xia XG (2011) Radon-Fourier transform for radar target detection, (I): generalized Doppler filter bank. IEEE Trans Aerosp Electron Syst 47(2):1186-1202. https://doi. org/10.1109/TAES.2011.5751251

11. Xu J, Yu J, Peng YN, Xia XG (2011) Radon-Fourier transform for radar target detection (II): blind speed sidelobe suppression. IEEE Trans Aerosp Electron Syst 47(4):2473-2489. https://doi. org/10.1109/TAES.2011.6034645

12. Yu J, Xu J, Peng YN, Xia XG (2012) Radon-Fourier transform for radar target detection (III): optimality and fast implementations. IEEE Trans Aerosp Electron Syst 48(2):991-1004. https:// doi.org/10.1109/TAES.2012.6178044

13. Rao X, Zhong T, Tao H, Xie J, Su J (2018) Improved axis rotation MTD algorithm and its analysis. Multidimens Syst Signal Process. https://doi.org/10.1007/s11045-018-0588-y

14. Perry RP, Dipietro RC, Fante R (1999) SAR imaging of moving targets. IEEE Trans Aerosp Electron Syst 35(1):188-200

15. Li H, Ma D, Wu R (2017) A low complexity algorithm for across range unit effect correction of the moving target via range frequency polynomial-phase transform. Digit Signal Process 62:176-186. https://doi.org/10.1016/j.dsp.2016.12.001

16. Xu J, Xia XG, Peng SB, Yu J, Peng YN, Qian LC (2012) Radar maneuvering target motion estimation based on generalized Radon-Fourier transform. IEEE Trans Signal Process 60(12):61906201. https://doi.org/10.1109/TSP.2012.2217137

17. Wu W, Wang GH, Sun JP (2018) Polynomial Radon-polynomial Fourier transform for near space hypersonic maneuvering target detection. IEEE Trans Aerosp Electron Syst 54(3):1306-1322. https://doi.org/10.1109/TAES.2017.2780658

18. Zhou F, Wu R, Xing M, Bao Z (2007) Approach for single channel SAR ground moving target imaging and motion parameter estimation. IET Radar Sonar Navig 1(1):59-66. https://doi. org/10.1049/iet-rsn:20060040

19. Kong L, Li X, Cui G, Yi W, Yang Y (2015) Coherent integration algorithm for a maneuvering target with high-order range migration. IEEE Trans Signal Process 63(17):4474-4486. https:// doi.org/10.1109/TSP.2015.2437844

20. Huang P, Liao G, Yang Z, Shu Y, Du W (2015) Approach for spacebased radar manoeuvring target detection and high-order motion parameter estimation. IET Radar Sonar Navig 9(6):732741. https://doi.org/10.1049/iet-rsn.2014.0192

21. Liu QH, Nguyen N, Tang XY (1998) Accurate algorithms for nonuniform fast forward and inverse Fourier transforms and their applications. In: Geoscience and remote sensing symposium proceedings, 1998. IGARSS ‘98. 1998 IEEE International, vol 281, pp 288-290

22. Liu QH, Nguyen $N$ (1998) An accurate algorithm for nonuniform fast Fourier transforms (NUFFT's). IEEE Microw Guided Wave Lett 8(1):18-20. https://doi.org/10.1109/75.650975

23. Nguyen N, Liu QH (1999) The regular Fourier matrices and nonuniform fast Fourier transforms. SIAM J Sci Comput 21(1):283-293

24. Fourmont $\mathrm{K}$ (2003) Non-equispaced fast Fourier transforms with applications to tomography. J Fourier Anal Appl 9(5):431-450

25. Hassanieh $H$, Indyk P, Katabi D, Price E (2012) Simple and practical algorithm for sparse Fourier transform. In: ACM-SIAM symposium on discrete algorithms, pp 1183-1194

Publisher's Note Springer Nature remains neutral with regard to jurisdictional claims in published maps and institutional affiliations. 